\title{
Treatment Patterns and Overall Survival Associated with First-Line Systemic Therapy for Patients with Advanced Non-Small Cell Lung Cancer
}

Michele M. Spence, PhD; Rita L. Hui, PharmD, MS; Jennifer T. Chang, PharmD, MPH; Joanne E. Schottinger, MD; Mirta Millares, PharmD, FCSHP, FASHP; and Nazia Rashid, PharmD, MS

\begin{abstract}
BACKGROUND: A variety of regimens are used as first-line treatment in patients with advanced non-small cell lung cancer (NSCLC), which may include combination regimens and single agents, depending on histology, molecular profile, and performance status.

OBJECTIVE: To describe the types of first-line therapies and compare overall survival between therapies used for patients with advanced NSCLC in an integrated health care system.
\end{abstract}

METHODS: This retrospective cohort study included patients aged 18 years or older from Kaiser Permanente California with a diagnosis of stage IIIB/IV NSCLC. First systemic treatment date occurred from January 1, 2008, through September 30, 2013. Overall survival was measured as the number of months from initial treatment until death, end of enrollment, or September 30, 2014. Treatment regimens were categorized into 6 mutually exclusive groups: platinum doublets; pemetrexed-based, bevacizumabbased, and pemetrexed + bevacizumab-based combinations; singlets; and tyrosine-kinase inhibitors (TKIs). Survival was compared using KaplanMeier curves and adjusted Cox proportional hazard models. Subgroup analyses were performed by age group and by nonsquamous histology.

RESULTS: Of 2,081 patients, approximately half $(52.3 \%)$ received platinum doublets, followed by TKIs (19.0\%), pemetrexed-based regimens $(13.4 \%)$, bevacizumab-regimens $(8.0 \%)$, singlets $(5.5 \%)$, and pemetrexed + bevacizumab-based combinations (1.8\%). Median survival was longest for pemetrexed + bevacizumab-based combinations ( 18.5 months), followed by bevacizumab-based regimens (14.5), TKIs (12.7), pemetrexed-based regimens (10.4), doublets (9.2), and singlets (5.3). There was a significantly reduced risk of mortality for pemetrexed + bevacizumab-based combinations $(\mathrm{HR}=0.64 ; 95 \% \mathrm{Cl}=0.42-0.94)$ and TKIs $(\mathrm{HR}=0.83 ; 95 \% \mathrm{Cl}=0.73-$ 0.94) compared with doublets. Singlets were associated with an increased risk of mortality $(\mathrm{HR}=1.50 ; 95 \% \mathrm{Cl}=1.22-1.84)$. Subgroup analysis among patients aged 65 years and over found no significant differences among treatment groups, with the exception of singlets, which were associated with an increased risk of mortality compared with doublets ( $\mathrm{HR}=1.51$; $95 \% \mathrm{Cl}=1.20-1.90)$. Among patients under aged 65 years, pemetrexed + bevacizumab-based combinations ( $\mathrm{HR}=0.36 ; 95 \% \mathrm{Cl}=0.21-0.64$ ) and TKIs $(\mathrm{HR}=0.76 ; 95 \% \mathrm{Cl}=0.59-0.97)$ were associated with a reduced risk of mortality, and singlets were associated with an increased risk ( $\mathrm{HR}=1.85$; 95\% $\mathrm{Cl}=1.17-2.92)$.

CONCLUSIONS: In this cohort of patients with advanced NSCLC, patients received a platinum agent with or without bevacizumab or pemetrexed, a $\mathrm{TKI}$, or a single agent. Younger patients (aged $<65$ years) receiving bevacizumab + pemetrexed-based combinations had a survival advantage over those receiving platinum doublets, and this finding merits further investigation. Younger patients receiving TKIs also had longer survival. Compared with platinum doublets, we found no survival advantage for older patients receiving bevacizumab or pemetrexed, which suggests that combination therapy of a platinum agent and taxane, such as carboplatin and paclitaxel, could be a reasonable option for older patients who are not candidates for targeted therapy.

\section{J Manag Care Spec Pharm. 2017;23(2):195-205}

Copyright $\odot 2017$, Academy of Managed Care Pharmacy. All rights reserved.

\section{What is already known about this subject}

Lung cancer is the second most commonly diagnosed cancer in the United States and the leading cause of cancer death among men and women.

About 80\%-85\% of lung cancers are non-small cell lung cancer (NSCLC). There are many possible treatment options for NSCLC, depending on histology, molecular targeting, oncogenic mutations, performance status, and other clinical factors.

\section{What this study adds}

This study examined differences in overall survival between firstline systemic regimens among patients with advanced NSCLC in a real-world setting.

Patients aged younger than 65 years who used bevacizumab+ pemetrexed-based combinations had a survival advantage over those using platinum doublets. Younger patients using tyrosinekinase inhibitors also had longer survival compared with platinum doublets.

None of the regimens produced a survival advantage over platinum doublets among patients aged 65 years or older.

A n estimated 224,390 patients were diagnosed with lung cancer in 2016, which represents $13.3 \%$ of all new newly diagnosed cancers and is the second most commonly diagnosed cancer. ${ }^{1}$ Non-small cell lung cancer (NSCLC) is the most common form of lung cancer and accounts for $85 \%$ of all lung cancer cases. ${ }^{2}$ The overall 5 -year survival rate for NSCLC is $17.7 \% .{ }^{1}$ Most patients with NSCLC are diagnosed at an advanced stage where survival rates continue to be dismally low. ${ }^{1}$ 
For years, platinum-based chemotherapy, such as carboplatin in combination with paclitaxel, has been the mainstay of first-line treatment of advanced NSCLC. However, newer regimens are now optimally defined by histology, molecular targeting of vascular endothelial growth factor (VEGF), or oncogenic driver mutations. The Eastern Cooperative Oncology Group's (ECOG) 4599 trial demonstrated that the addition of bevacizumab, a monoclonal antibody against VEGF, to carboplatin and paclitaxel improved overall survival by 2 months compared with carboplatin and paclitaxel alone. ${ }^{3}$ This trial was conducted among patients with nonsquamous histology, and a subsequent analysis by age found that elderly patients did not benefit from the addition of bevacizumab and had increased side effects. ${ }^{4}$ Retrospective studies have found similar results, although 1 study reported longer survival and fewer hospitalizations among a cohort of Medicare patients. ${ }^{5-7}$ In a secondary analysis of the ECOG 4599 and PointBreak trials, a survival benefit associated with the addition of bevacizumab was found for patients aged less than 75 years, but not for those aged 75 years or older. ${ }^{8}$

The National Comprehensive Cancer Network (NCCN) Guidelines Panel endorses broader molecular testing for epidermal growth factor receptor (EFGR) mutation and the anaplastic lymphoma kinase (ALK) gene. ${ }^{9}$ For patients that are not positive for either test and have a good performance status of 0 or 1 , two-drug chemotherapy is preferred. The NCCN guidelines note that many drugs are useful for stage IV NSCLC, including platinum agents such as carboplatin and cisplatin; taxanes such as paclitaxel and docetaxel; and vinorelbine, etoposide, pemetrexed, and gemcitabine. Bevacizumab may be added if the NSCLC is of nonsquamous histology, and the patient has no recent history of hemoptysis. Pemetrexed, an antifolate agent, is now one of the most frequently used chemotherapies for nonsquamous NSCLC. A phase III study showed that overall survival was better with cisplatin + pemetrexed than with cisplatin + gemcitabine among patients with nonsquamous NSCLC, with fewer grade 3 or 4 drug-related toxicities. ${ }^{10}$ Consequently, many patients now receive pemetrexed-based regimens rather than taxanes because of better tolerability. Pemetrexed is not indicated for the treatment of squamous cell NSCLC. For patients with squamous cell NSCLC and good performance status (and are not candidates for targeted therapy), the NCCN recommends doublet regimens without pemetrexed or bevacizumab. There is superior efficacy for cisplatin + gemcitabine in comparison with cisplatin + pemetrexed for patients with squamous cell NSCLC. ${ }^{10}$ For patients with poor performance status, the NCCN recommends singleagent chemotherapy or platinum-based doublets. Single-agent chemotherapy is often used because of concerns about toxicity. Best supportive palliative care is recommended for patients with a performance status of 3 or 4 .
Oral tyrosine-kinase inhibitors (TKIs) include erlotinib, gefitinib, afatinib, crizotinib, ceritinib, and alectinib. The NCCN recommends erlotinib, gefitinib, or afatinib for patients with metastatic nonsquamous NSCLC who are EGFR positive, regardless of their performance status. Quality of life is improved for patients using TKIs compared with chemotherapy, and they have fewer side effects. ${ }^{9}$ Crizotinib is recommended for patients who are positive for the ALK gene rearrangement.

Most first-line systemic therapies for NSCLC have been shown to produce 1-year survival rates of 30\%-40\%. ${ }^{9}$ However, integrating the data from numerous trials and selecting the most appropriate therapy for patients remains challenging, especially for patients with nonsquamous histology and no known oncogenic driver mutations. Few studies have used real-world data to compare the clinical outcomes associated with more than 2 or 3 different first-line regimens. This study aims to describe the types of first-line therapies and compare overall survival between therapies used for patients with advanced NSCLC in an integrated health care system.

\section{Methods}

\section{Data Sources}

A retrospective cohort design was used to compare treatment regimens among patients from Kaiser Permanente (KP) California, an integrated health care system that serves over 8 million members in California. ${ }^{11}$ We identified all patients with a diagnosis of stage IIIB or stage IV NSCLC from the KP Southern California Cancer Registry and the KP Northern California Cancer Registry. Both registries document invasive and in situ cancer, including all solid tumors and systemic malignancies. Cases are reported to the California Cancer Registry and the National Surveillance, Epidemiology and EndResults Program. ${ }^{1}$ Pharmacy and chemotherapy infusion data were extracted from electronic health records and databases, as were coded inpatient and outpatient encounters. Codes were based on the International Classification of Diseases, Ninth Revision, Clinical Modification (ICD-9-CM) system. Demographic and enrollment data were taken from administrative membership databases. Death data were derived from the cancer registry, membership data, and state death certificates from the Social Security Administration. This study was approved by the institutional review boards of KP Southern California (KPSC) and KP Northern California (KPNC).

\section{Study Sample}

Patients had to have the initial treatment for stage IIIB or stage IV NSCLC after the diagnosis of NSCLC from January 1 , 2008, through September 30, 2013, and were followed until death, disenrollment from the health plan, or September 30, 2014, whichever came first. The initial first-line treatment was defined as the index date. Patients were also required to have continuous health plan enrollment and a drug benefit at least 
12 months before the index date. Patients were excluded if they were aged younger than 18 years, died within 1 month of the index date, or initiated treatment more than 120 days after diagnosis of NSCLC. Based on the 1-year survival rates of 30\%$40 \%$ for patients using first-line systemic therapies for NSCLC, we estimated that we would need about 350 patients in each treatment group to detect a $10 \%$ difference in survival between groups at $80 \%$ power and an alpha level of 0.05 .

\section{Identification of First-Line Treatment Groups}

First-line treatment was defined as initiation of systemic or oral chemotherapy within 120 days of NSCLC diagnosis. We identified all the initial treatment regimens and then grouped the most frequently used agents into 6 categories. Because we initially identified over 28 different regimens for first-line treatment, we decided to group them into similar categories that were clinically meaningful and that allowed analysis. The most frequently used agents were categorized in these 6 groups: (1) platinum doublets, which included any platinum chemotherapy in combination with a single agent, excluding pemetrexed and bevacizumab; (2) pemetrexed-based regimen using pemetrexed alone or in combination with platinum chemotherapy; (3) bevacizumab-based regimen, which included bevacizumab alone or in combination with a single agent or platinum chemotherapy; (4) pemetrexed+bevacizumab, which included pemetrexed and bevacizumab with or without a platinum agent; (5) single nonplatinum agents; and (6) TKIs (Appendix A, available in online article).

\section{Outcomes}

Overall survival was the primary outcome, defined as the number of months from the start of first-line therapy until death. Death data were captured until September 30, 2014. Patients who were disenrolled from the health plan or who were alive at the end of the study period were censored. We also described the most common chemotherapy-related adverse events, which were identified from package inserts and classified using ICD9-CM diagnosis codes and diagnosis-related groups after the initiation of chemotherapy (Appendix B, available in online article).

\section{Baseline Covariates}

Demographic and clinical data included age at index date, gender, race, geographic region (KPSC or KPNC), stage of tumor at diagnosis, histology, comorbidities, and smoking status. Race was identified from the electronic medical record and was categorized as non-Hispanic white, non-Hispanic black, Asian/ Pacific Islander, Hispanic, or other/unknown. Tumor stage IIIB or IV was defined using the American Joint Committee on Cancer from the cancer registry, as was squamous or nonsquamous NSCLC. We measured the comorbidity level of patients using the Deyo modification of the Charlson Comorbidity Index, which excludes cancer diagnoses from the score. ${ }^{12}$
This modification uses inpatient and outpatient diagnosis 12 months before the index date (excluding the first month before the index date) to calculate a score for each patient in the study. Scores can range from 0 to 15 , with higher scores indicating greater morbidity. Smoking status was identified from patients' medical records and classified as current smoker, past smoker, or never smoked.

\section{Statistical Analysis}

Analyses were performed by comparing each of the 5 treatment groups with the platinum doublets group (reference group). Baseline characteristics were compared using the chi-square test or Fisher's exact text for differences in percentages and t-tests for differences in means. The Kaplan-Meier method was used to characterize the contour of survival over time and estimate differences in median survival between the 6 groups using the log-rank test. We conducted unadjusted and adjusted multivariable Cox proportional hazards models controlling for demographic and clinical characteristics to examine the effect of the 5 treatment regimens on doublets for overall survival and to examine patient characteristics related to survival. The models were adjusted for age, sex, race, region, stage, histology, Charlson Comorbidity Index, smoking, and subsequent therapy. The proportional hazards assumption was tested during the modeling process by adding an interaction term for each covariate by time. We also conducted subgroup analyses by age group (65 years or over and under 65 years) and by nonsquamous histology.

Because this was a retrospective study, there is the potential for bias caused by confounders that can predispose patients to be selected for a particular treatment group. To minimize this potential bias, we used a propensity score approach to balance any systematic differences in observed confounders between the treatment groups. This propensity score is the conditional probability of each patient receiving a particular treatment based on pretreatment variables. Multivariate logistic regression was employed to calculate the propensity scores for each treatment group. We then used the inverse probability of treatment weight (IPTW) to weight the observations for better control of confounding. These weights were then normalized to reflect the sample size for each of the treatment groups. Chisquare tests and $\mathrm{P}$ values were used to assess whether baseline characteristics were balanced across the 6 treatment groups after weighting. Finally, Cox proportional hazards models were rerun using the IPTW normalized weights to assess overall survival. Statistical significance was set at $P<0.05$, and all tests were 2-tailed. The SAS statistical package, version 9.2, was used for all analysis (SAS Institute, Cary, NC).

\section{Results}

There were 2,081 patients in the cohort. Approximately half (52.3\%) were using platinum doublets, followed by TKIs (19.0\%), 
Treatment Patterns and Overall Survival Associated with First-Line Systemic Therapy for Patients with Advanced Non-Small Cell Lung Cancer

TABLE 1 Demographic and Clinical Characteristics of NSCLC Cohort by Treatment Group

\begin{tabular}{|c|c|c|c|c|c|c|c|c|c|c|c|}
\hline Characteristic & $\begin{array}{l}\text { Doublets } \\
n=1,088\end{array}$ & $\begin{array}{c}\text { Pem-based } \\
\mathbf{n}=279\end{array}$ & $P$ Value ${ }^{a}$ & $\begin{array}{c}\text { Pem }+ \\
\text { Bev-based } \\
\mathbf{n}=38\end{array}$ & $P$ Value & $\begin{array}{c}\text { Bev-based } \\
\mathrm{n}=167\end{array}$ & $P$ Value & $\begin{array}{c}\text { Singlets } \\
\mathrm{n}=114\end{array}$ & $P$ Value & $\begin{array}{c}\text { TKI } \\
\mathbf{n}=395\end{array}$ & $P$ Value \\
\hline Age, mean (SD) & $66.3( \pm 9.5)$ & $66.3( \pm 10.3)$ & 0.924 & $60.7( \pm 9.0)$ & $<0.001$ & $64.4( \pm 9.6)$ & 0.019 & $73.1( \pm 9.9)$ & $<0.001$ & $69.6( \pm 12.0)$ & $<0.001$ \\
\hline \multicolumn{12}{|l|}{ Gender, n (\%) } \\
\hline Male & 597 (54.9) & $145(52.0)$ & 0.386 & $20(52.6)$ & 0.785 & $95(56.9)$ & 0.626 & $63(55.3)$ & 0.936 & $144(36.5)$ & $<0.001$ \\
\hline Female & $491(45.1)$ & $134(48.0)$ & & $18(47.4)$ & & $72(43.1)$ & & $51(44.7)$ & & $251(63.5)$ & \\
\hline \multicolumn{12}{|l|}{ Race, n (\%) } \\
\hline White & $734(67.5)$ & $172(61.7)$ & 0.254 & $21(55.3)$ & 0.089 & $106(63.5)$ & 0.024 & $81(71.1)$ & 0.563 & $209(52.9)$ & $<0.001$ \\
\hline Black & $135(12.4)$ & $42(15.1)$ & & $6(15.8)$ & & $16 \quad(9.6)$ & & $14(12.3)$ & & $27 \quad(6.8)$ & \\
\hline Asian & $113(10.4)$ & $35(12.5)$ & & $9(23.7)$ & & $30(18.0)$ & & $13(11.4)$ & & $120(30.4)$ & \\
\hline Hispanic & $94 \quad(8.6)$ & $29(10.4)$ & & $2 \quad(5.3)$ & & $11 \quad(6.6)$ & & $6 \quad(5.3)$ & & $\begin{array}{ll}36 & (9.1)\end{array}$ & \\
\hline Other/unknown & $12 \quad(1.1)$ & $1 \quad(0.4)$ & & $0(0.00)$ & & $4 \quad(2.4)$ & & $0(0.00)$ & & $3 \quad(0.8)$ & \\
\hline \multicolumn{12}{|l|}{ Region, n (\%) } \\
\hline South & $378(34.7)$ & $127(45.5)$ & 0.001 & $21(55.3)$ & 0.009 & $72(43.1)$ & 0.036 & $39(34.2)$ & 0.910 & $154(39.0)$ & 0.132 \\
\hline North & $710(65.3)$ & $152(54.5)$ & & $17(44.7)$ & & $95(56.9)$ & & $75(65.8)$ & & $241(61.0)$ & \\
\hline \multicolumn{12}{|l|}{ AJCC stage, n (\%) } \\
\hline IIIB & $229(21.1)$ & $20 \quad(7.2)$ & $<0.001$ & $4(10.5)$ & 0.116 & $20(12.0)$ & 0.006 & $17(14.9)$ & 0.122 & $41(10.4)$ & $<0.001$ \\
\hline IV & $859(79.0)$ & $259(92.8)$ & & $34(89.5)$ & & $147(88.0)$ & & $97(85.1)$ & & $354(89.6)$ & \\
\hline \multicolumn{12}{|l|}{ Histology, n (\%) } \\
\hline Squamous & $272(25.0)$ & $2 \quad(0.7)$ & $<0.001$ & $1 \quad(2.6)$ & 0.002 & $4 \quad(2.4)$ & $<0.001$ & $19(16.7)$ & 0.048 & $16 \quad(4.1)$ & $<0.001$ \\
\hline Nonsquamous & $816(75.0)$ & 277 (99.3) & & $37(97.4)$ & & $163(97.6)$ & & $95(83.3)$ & & $379(96.0)$ & \\
\hline \multicolumn{12}{|c|}{ Subsequent therapy, n (\%) } \\
\hline No & $538(49.5)$ & $113(40.5)$ & 0.008 & $14(36.8)$ & 0.127 & $55(32.9)$ & $<0.001$ & $65(57.0)$ & 0.124 & $254(64.3)$ & $<0.001$ \\
\hline Yes & $550(50.6)$ & $166(59.5)$ & & $24(63.2)$ & & $112(67.1)$ & & $49(43.0)$ & & $141(35.7)$ & \\
\hline $\begin{array}{l}\text { Charlson score, } \\
\text { mean (SD) }\end{array}$ & $1.06( \pm 1.5)$ & $0.98( \pm 1.5)$ & 0.420 & $0.79( \pm 1.5)$ & 0.266 & $0.83( \pm 1.4)$ & 0.059 & $1.47( \pm 1.8)$ & 0.020 & $1.11( \pm 1.6)$ & 0.622 \\
\hline \multicolumn{12}{|c|}{ Smoking status, n (\%) } \\
\hline Current smoker & $133(12.2)$ & $20 \quad(7.2)$ & 0.005 & $1 \quad(2.6)$ & $<0.001$ & $11 \quad(6.6)$ & $<0.001$ & $8 \quad(7.0)$ & 0.160 & $19 \quad(4.8)$ & $<0.001$ \\
\hline Never smoked & $161(14.8)$ & $59(21.2)$ & & $15(39.5)$ & & $43(25.8)$ & & $14(12.3)$ & & $198(50.1)$ & \\
\hline Past smoker & $794(73.0)$ & $200(71.7)$ & & $22(57.9)$ & & $113(67.7)$ & & $92(80.7)$ & & $178(45.1)$ & \\
\hline
\end{tabular}

pemetrexed-based regimens (13.4\%), bevacizumab-based regimens $(8.0 \%)$, singlets $(5.5 \%)$, and pemetrexed + bevacizumabbased combinations (1.8\%). Appendix A describes the specific regimens and agents that were used in each of the 6 treatment groups. The most common regimen was carboplatin + paclitaxel, used by 739 (35.5\%) patients. There were 395 (19.0\%) patients receiving TKIs; erlotinib was the only TKI agent used.

There were significant differences between the treatment groups in demographic and clinical characteristics (Table 1). Compared with doublets, patients receiving bevacizumabbased or pemetrexed + bevacizumab-based regimens were younger, and those taking singlets were older and had significantly higher Charlson comorbidity scores. Those receiving a TKI were more likely to be female, Asian, diagnosed at stage IV, had nonsquamous histology, and had never smoked. Patients on pemetrexed, bevacizumab, or pemetrexed+bevacizumab combinations were also more likely to have never smoked and were more likely to have nonsquamous histology than those on doublets. Patients receiving pemetrexed-based or bevacizumabbased regimens were more likely to go on to subsequent therapy compared with platinum doublets, whereas those receiving a TKI were less likely to receive subsequent therapy.

Kaplan-Meier curves (Figure 1) showed an early separation for the pemetrexed + bevacizumab-based group, whose median survival was 18.5 months, followed by the bevacizumab-based group with a median survival of 14.5 months, TKIs (12.7 months), pemetrexed-based regimens (10.4 months), and doublets (9.2 months). Singlets had the shortest median survival of 5.3 months

Propensity score adjusted models in Table 2 indicate that pemetrexed+bevacizumab-based regimens were associated with a reduced risk of mortality (hazard ratio $[\mathrm{HR}]=0.63 ; 95 \%$ confidence interval $[\mathrm{CI}]=0.42-0.94 ; P=0.023$ ), as were TKIs $(\mathrm{HR}=0.83 ; 95 \% \mathrm{CI}=0.73-0.94 ; P=0.004)$. Singlets were associated with an increased risk ( $\mathrm{HR}=1.50 ; 95 \% \mathrm{CI}=1.22-1.84$; $P<0.001)$. Patient characteristics related to an increased risk 


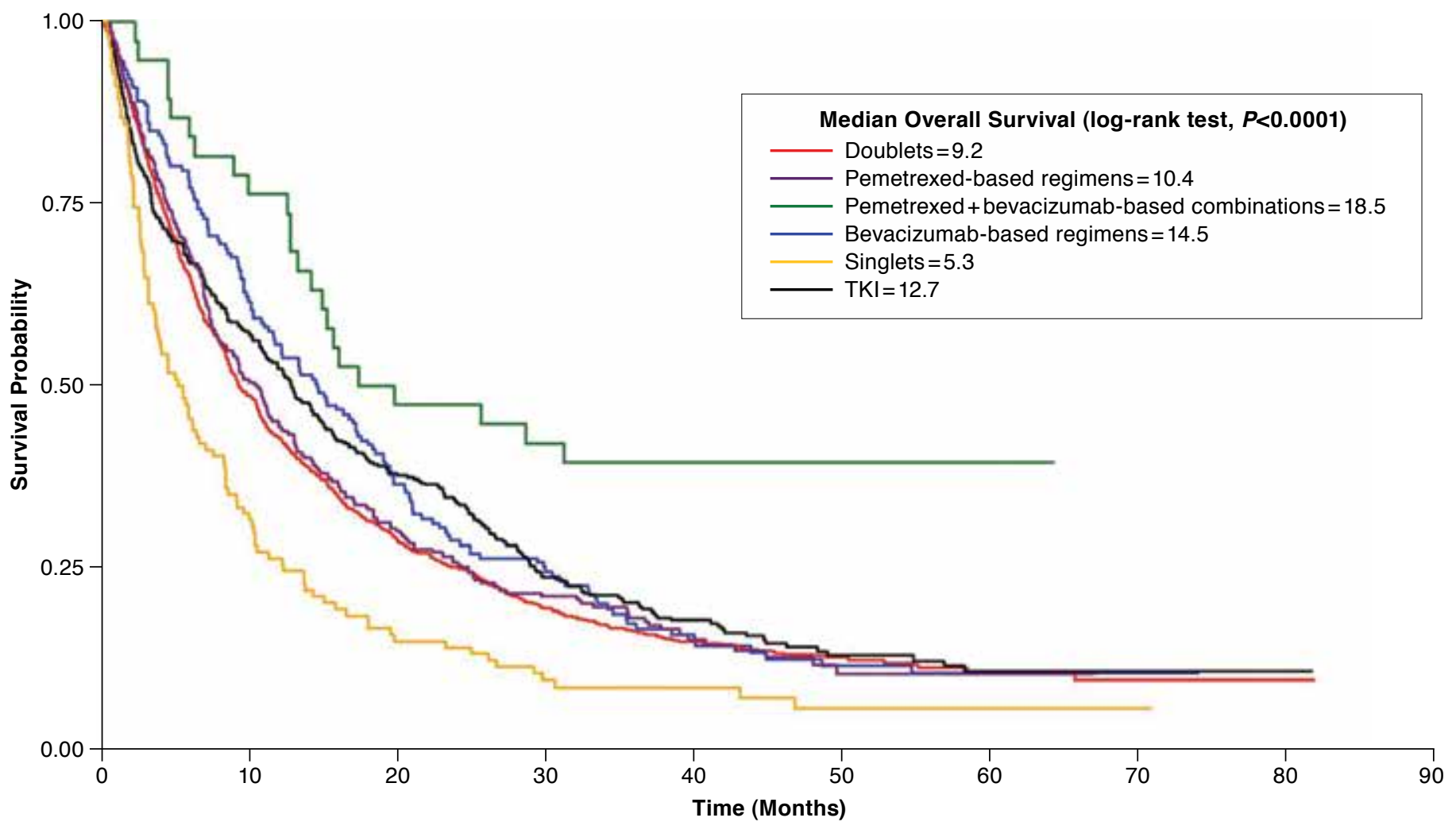

OS= overall survival; $T K I=$ tyrosine-kinase inhibitor.

of mortality included a diagnosis of stage IV ( $\mathrm{HR}=1.86$; 95\% $\mathrm{CI}=1.62-2.14 ; \quad P<0.001)$, squamous histology $(\mathrm{HR}=1.19$; 95\% CI $=1.04-1.35 ; P=0.010$ ), and higher Charlson comorbidity score $(\mathrm{HR}=1.04 ; 95 \% \mathrm{CI}=1.01-1.07 ; P=0.021)$. Subsequent treatment for NSCLC was associated with a reduced risk of mortality (HR=0.44; 95\% CI =0.40-0.49; $P<0.001)$.

Subgroup analysis for patients aged less than 65 years revealed similar results as the model for all ages (Table 3). Pemetrexed + bevacizumab-based combinations ( $\mathrm{HR}=0.36$; 95\% CI $=0.21-0.64 ; \quad P<0.001)$ and TKIs $(\mathrm{HR}=0.76 ; 95 \%$ $\mathrm{CI}=0.59-0.97 ; P=0.030)$ were associated with a reduced risk of mortality, while singlets had an increased risk ( $\mathrm{HR}=1.85 ; 95 \%$ $\mathrm{CI}=1.17-2.92 ; \mathrm{P}=0.009$ ). For patients aged 65 years or older, however, only singlets were significantly associated with an increased risk of mortality ( $\mathrm{HR}=1.51 ; 95 \% \mathrm{CI}=1.20-1.90$; $P<0.001)$. The subgroup analysis of patients with nonsquamous histology also showed a reduced risk of mortality for the pemetrexed + bevacizumab-based group ( $\mathrm{HR}=0.47 ; 95 \%$ $\mathrm{CI}=0.30-0.72 ; P<0.001)$ and TKIs $(\mathrm{HR}=0.83 ; 95 \% \mathrm{CI}=0.72$ $0.96 ; P=0.013)$ and increased risk for singlets $(H R=1.40 ; 95 \%$ $\mathrm{CI}=1.11-1.75 ; P=0.004)$.
Table 4 shows the distribution of adverse events by treatment group. Over $76 \%$ of patients taking doublets experienced at least 1 adverse event, with the majority having anemia (44.1\%). Compared with doublets, only TKIs had a significantly lower rate of adverse events $(66.3 \% ; P<0.001)$. Patients taking TKIs had lower rates of anemia, bone marrow depression, leukopenia, neutropenia, thrombocytopenia, and venous and arterial thrombosis. However, they had a higher rate of skin rash compared with doublets ( $14.9 \%$ vs. $9.0 \% ; P=0.001)$. Patients using pemetrexed-based regimens had a higher rate of pulmonary embolism than those taking doublets ( $13.6 \%$ vs. $6.5 \% ; P=0.002)$

\section{Discussion}

In this study, a majority of patients with advanced NSCLC received platinum doublets. Carboplatin-based doublets ( $n=910 ; 43.7 \%$ ) were more commonly used than cisplatinbased doublets ( $n=178 ; 8.5 \%$ ), perhaps because of concerns about greater toxicity associated with cisplatin. ${ }^{13}$ The most common first-line regimen was carboplatin + paclitaxel, which is similar to a study conducted at 8 NCCN institutions, where 
Treatment Patterns and Overall Survival Associated with First-Line Systemic Therapy for Patients with Advanced Non-Small Cell Lung Cancer

TABLE 2 Hazard Ratios for Overall Survival for Study Treatment Groups

\begin{tabular}{|c|c|c|c|c|c|c|c|c|c|}
\hline \multirow[b]{2}{*}{ Characteristic } & \multicolumn{3}{|c|}{ Unadjusted } & \multicolumn{3}{|c|}{ Adjusted } & \multicolumn{3}{|c|}{ Propensity Score Adjusted } \\
\hline & HR & $95 \% \mathrm{CI}$ & $P$ Value & HR & $95 \% \mathrm{CI}$ & $P$ Value & HR & $95 \%$ CI & $P$ Value \\
\hline \multicolumn{10}{|l|}{ Treatment group } \\
\hline Doublets & 1.0 [Ref] & 1.0 [Ref] & - & 1.0 [Ref] & 1.0 [Ref] & - & 1.0 [Ref] & 1.0 [Ref] & - \\
\hline Pem-based & 0.96 & $0.84-1.11$ & 0.602 & 0.98 & $0.84-1.13$ & 0.754 & 1.04 & $0.90-1.20$ & 0.590 \\
\hline Pem+Bev-based & 0.48 & $0.32-0.72$ & 0.001 & 0.50 & $0.33-0.76$ & 0.001 & 0.63 & $0.42-0.94$ & 0.023 \\
\hline Bev-based & 0.85 & $0.71-1.01$ & 0.066 & 0.97 & $0.81-1.16$ & 0.741 & 1.04 & $0.88-1.24$ & 0.653 \\
\hline Singlets & 1.55 & $1.26-1.89$ & $<0.001$ & 1.53 & $1.25-1.87$ & $<0.001$ & 1.50 & $1.22-1.84$ & $<0.001$ \\
\hline TKI & 0.88 & $0.78-1.00$ & 0.045 & 0.85 & $0.74-0.98$ & 0.028 & 0.83 & $0.73-0.94$ & 0.004 \\
\hline \multicolumn{10}{|l|}{ Age, years } \\
\hline$<65$ & & & & 1.0 [Ref] & $1.0[\operatorname{Ref}]$ & - & 1.0 [Ref] & $1.0[\operatorname{Ref}]$ & - \\
\hline$\geq 65$ & & & & 1.00 & 0.91-1.11 & 0.947 & 0.98 & $0.89-1.08$ & 0.674 \\
\hline \multicolumn{10}{|l|}{ Gender } \\
\hline Female & & & & 1.0 [Ref] & 1.0 [Ref] & - & 1.0 [Ref] & 1.0 [Ref] & - \\
\hline Male & & & & 1.11 & $1.00-1.22$ & 0.045 & 1.09 & $0.99-1.20$ & 0.071 \\
\hline \multicolumn{10}{|l|}{ Race } \\
\hline White & & & & 1.0 [Ref] & $1.0[\operatorname{Ref}]$ & - & 1.0 [Ref] & $1.0[\mathrm{Ref}]$ & - \\
\hline Black & & & & 0.95 & $0.82-1.11$ & 0.538 & 0.90 & $0.77-1.04$ & 0.146 \\
\hline Asian & & & & 0.89 & $0.77-1.03$ & 0.131 & 0.92 & $0.80-1.06$ & 0.231 \\
\hline Hispanic & & & & 0.89 & $0.75-1.06$ & 0.202 & 0.88 & $0.74-1.04$ & 0.133 \\
\hline Other/unknown & & & & 1.05 & $0.66-1.65$ & 0.843 & 1.03 & $0.65-1.63$ & 0.891 \\
\hline \multicolumn{10}{|l|}{ Region } \\
\hline North & & & & 1.0 [Ref] & 1.0 [Ref] & - & 1.0 [Ref] & $1.0[\mathrm{Ref}]$ & - \\
\hline South & & & & 1.01 & $0.92-1.12$ & 0.803 & 1.09 & $0.99-1.20$ & 0.091 \\
\hline \multicolumn{10}{|l|}{ AJCC stage } \\
\hline IIIB & & & & $1.0[\mathrm{Ref}]$ & 1.0 [Ref] & - & $1.0[\operatorname{Ref}]$ & 1.0 [Ref] & - \\
\hline IV & & & & 1.78 & $1.56-2.04$ & $<0.001$ & 1.86 & $1.62-2.14$ & $<0.001$ \\
\hline \multicolumn{10}{|l|}{ Histology } \\
\hline Nonsquamous & & & & $1.0[\operatorname{Ref}]$ & $1.0[\operatorname{Ref}]$ & - & $1.0[\mathrm{Ref}]$ & $1.0[\mathrm{Ref}]$ & - \\
\hline Squamous & & & & 1.07 & $0.94-1.23$ & 0.319 & 1.19 & $1.04-1.35$ & 0.010 \\
\hline \multicolumn{10}{|c|}{ Subsequent therapy } \\
\hline No & & & & 1.0 [Ref] & 1.0 [Ref] & - & 1.0 [Ref] & 1.0 [Ref] & - \\
\hline Yes & & & & 0.50 & $0.45-0.55$ & $<0.001$ & 0.44 & $0.40-0.49$ & $<0.001$ \\
\hline Charlson score & & & & 1.04 & $1.01-1.07$ & 0.019 & 1.04 & $1.01-1.07$ & 0.021 \\
\hline \multicolumn{10}{|l|}{ Smoking status } \\
\hline Past & & & & 1.0 [Ref] & 1.0 [Ref] & - & 1.0 [Ref] & 1.0 [Ref] & - \\
\hline Current & & & & 1.13 & $0.96-1.33$ & 0.129 & 1.09 & 0.93-1.29 & 0.280 \\
\hline Never & & & & 0.89 & $0.78-1.01$ & 0.069 & 0.93 & $0.82-1.05$ & 0.249 \\
\hline
\end{tabular}

the most common regimen among off-trial patients was also carboplatin + paclitaxel. ${ }^{14}$ Pemetrexed combined with either carboplatin or cisplatin was also frequently used in both the NCCN study and our study. Among regimens containing bevacizumab, the combination of carboplatin + paclitaxel + bevacizumab was the most frequently used, although there was slightly lower use in our cohort (7\%) compared with the NCCN institutions (11\%). Another similarity found was that regimens containing pemetrexed and bevacizumab were not commonly used: $2 \%$ in our study and $4 \%$ in the NCCN study. Erlotinib was also the only TKI used, and use was higher in our study (19\%) compared with the NCCN study (13\%), possibly because of the longer and more recent time frame of our study, which took place 2008-2013, whereas the NCCN study time frame was 2006-2009. Singlets were used in about 5\% of both cohorts, most likely among patients with poor prognosis.

Patient characteristics that were associated with the various regimens appear to be consistent with NCCN guidelines. Two drug regimens are preferred, and patients with squamous histology do not benefit from either bevacizumab or pemetrexed. ${ }^{9}$ Most patients in our cohort were on 2 drug regimens, and very few patients with squamous NSCLC were taking any combination with bevacizumab or pemetrexed. Those with squamous histology in our cohort were also less likely to be using a TKI 


\begin{tabular}{|c|c|c|c|c|}
\hline Subgroup & Number & $\begin{array}{c}\text { Adjusted } \\
\text { HR }^{\mathrm{a}}\end{array}$ & $95 \% \mathrm{CI}$ & $P$ Value \\
\hline \multicolumn{5}{|c|}{ Age under 65 years } \\
\hline Doublets & 449 & 1.0 [Ref] & - & - \\
\hline Pem-based & 123 & 0.84 & $0.67-1.07$ & 0.157 \\
\hline Pem + Bev-based & 26 & 0.36 & $0.21-0.64$ & $<0.001$ \\
\hline Bev-based & 82 & 0.87 & $0.67-1.15$ & 0.329 \\
\hline Singlets & 21 & 1.85 & $1.17-2.92$ & 0.009 \\
\hline TKI & 131 & 0.76 & $0.59-0.97$ & 0.030 \\
\hline \multicolumn{5}{|c|}{ Age 65 years and older } \\
\hline Doublets & 639 & 1.0 [Ref] & - & - \\
\hline Pem-based & 156 & 1.07 & $0.88-1.30$ & 0.475 \\
\hline Pem + Bev-based & 12 & 0.83 & $0.44-1.55$ & 0.550 \\
\hline Bev-based & 85 & 1.05 & $0.82-1.34$ & 0.705 \\
\hline Singlets & 93 & 1.51 & $1.20-1.90$ & $<0.001$ \\
\hline TKI & 264 & 0.91 & $0.76-1.08$ & 0.265 \\
\hline \multicolumn{5}{|c|}{ Nonsquamous histology } \\
\hline Doublets & 816 & 1.0 [Ref] & - & - \\
\hline Pem-based & 277 & 0.95 & $0.81-1.10$ & 0.468 \\
\hline Pem + Bev-based & 37 & 0.47 & $0.30-0.72$ & $<0.001$ \\
\hline Bev-based & 163 & 0.95 & $0.79-1.15$ & 0.603 \\
\hline Singlets & 95 & 1.40 & $1.11-1.75$ & 0.004 \\
\hline TKI & 379 & 0.83 & $0.72-0.96$ & 0.013 \\
\hline \multicolumn{5}{|c|}{$\begin{array}{l}\text { aAdjusted for age, sex, race, region, stage, histology, Charlson Comorbidity Index, } \\
\text { smoking, and subsequent therapy. } \\
\text { Bev=bevacizumab; } C I=\text { confidence interval; } H R=\text { hazard ratio; } P \text { Pem = pemetrexed; } \\
\text { Ref = reference group; } T K I=\text { tyrosine-kinase inhibitor. }\end{array}$} \\
\hline
\end{tabular}

(erlotinib), perhaps because patients with squamous cell carcinoma are unlikely to have sensitizing EGFR mutations. ${ }^{15}$ Although we were not able to determine EGFR mutation testing results from our databases, we found that patients on TKIs in our cohort were more likely to be never smokers, female, or Asian-3 characteristics that are associated with a higher prevalence of EGFR mutations. ${ }^{16}$ Patients who were given singlet chemotherapy were older and had higher Charlson comorbidity scores, which may indicate that these patients had higher performance status. The NCCN recommends that single-agent chemotherapy may be appropriate in select patients who have a performance status of 2 and are not candidates for targeted therapies.

We found a decreased risk of mortality associated with pemetrexed + bevacizumab-based regimens, mainly in combination with cisplatin and carboplatin. Although this was a small group $(n=34)$, the results also held for the subgroup analysis among those patients aged less than 65 years and those with nonsquamous NSCLC. The PointBreak trial found that pemetrexed + bevacizumab and carboplatin followed by pemetrexed + bevacizumab maintenance therapy resulted in significantly longer progression-free survival compared with paclitaxel + bevacizumab + carboplatin, but with no difference in overall survival. ${ }^{17}$ Similarly, the AVAPERL trial compared pemetrexed + bevacizumab + cisplatin as first-line therapy, followed by bevacizumab with or without pemetrexed and found prolonged progression-free survival for the pemetrexed + bevacizumab group, but the study was not powered to show a statistically significant improvement in overall survival. ${ }^{18}$ The NCCN guidelines do have bevacizumab + pemetrexed with either carboplatin or cisplatin regimens listed as possible first-line options for selected patients, but it is not a category 1 recommendation. Our results must be interpreted with caution, but an explanation may be that these were patients with good performance status who were enrolled in a clinical trial. A post hoc chart review of the 34 patients revealed that none were participating in a clinical trial, and the prescribing oncologists noted that the patients had excellent performance status and would be able to tolerate the regimen. In addition, many of the prescribers were early adopters of this regimen based on emerging data and initial results from the PointBreak trial. ${ }^{19}$ This result highlights the importance of early research results and demonstrates the incorporation of new evidence into clinical practice.

Patient characteristics associated with reduced overall survival included stage IV, squamous histology, and higher Charlson comorbidity scores. Patients diagnosed at later stages and those who were sicker and had a greater burden of comorbidities had a poorer survival outlook. We also found that patients moving to subsequent therapy after first-line treatment had improved survival. Although we could not tell whether patients were put on continuous or switch maintenance, longer survival among these patients may be because of a "survivor effect," in other words, they survived long enough to receive maintenance therapy. We conducted a sensitivity analysis among patients who did not use subsequent therapy $(n=1,039)$ and found similar results for most treatment groups compared with doublets, with the exception that the TKI group no longer had a significantly decreased risk of mortality (pemetrexedbased regimens $[\mathrm{HR}=0.89 ; 95 \% \mathrm{CI}=0.71-1.12 ; \quad P=0.318]$; pemetrexed + bevacizumab-based combinations $[\mathrm{HR}=0.53$; 95\% CI $=0.282-0.99 ; P=0.053$ ]; bevacizumab-based regimens $[\mathrm{HR}=1.29 ; 95 \% \mathrm{CI}=0.96-1.73 ; P=0.092]$; singlets $[\mathrm{HR}=1.61$; 95\% CI $=1.23-2.10 ; P \leq 0.001]$; and TKIs $[\mathrm{HR}=1.01 ; 95 \%$ $\mathrm{CI}=0.84-1.21 ; P=0.931]$ ).

Among patients aged 65 years and older, the subgroup analysis showed no survival advantage for any of the treatment groups compared with platinum doublets. This is congruent with previous findings; for example, the ECOG 4599 trial found no advantage for the use of bevacizumab in this population, as did several retrospective studies. ${ }^{4-6,8}$ The NCCN guidelines, however, do not make specific recommendations by age; performance status is a more important risk factor than age alone. Phase III trials have shown improved overall survival in first-line treatment for elderly patients taking platinum doublets compared with singlets. For example, 2 trials showed that 
Treatment Patterns and Overall Survival Associated with First-Line

Systemic Therapy for Patients with Advanced Non-Small Cell Lung Cancer

TABLE 4 Adverse Events by Treatment Group

\begin{tabular}{|c|c|c|c|c|c|c|c|c|c|c|c|}
\hline Adverse Drug Event & $\begin{array}{c}\text { Doublets } \\
\mathrm{n}=1,088 \\
\mathrm{n}(\%)\end{array}$ & $\begin{array}{c}\text { Pem-based } \\
\mathrm{n}=279 \\
\mathrm{n}(\%)\end{array}$ & $P$ Value & $\begin{array}{c}\text { Pem }+ \text { Bev- } \\
\text { based } \\
\mathbf{n}=38 \\
\mathbf{n}(\%) \\
\end{array}$ & $P$ Value & $\begin{array}{c}\text { Bev-based } \\
\mathrm{n}=167 \\
\mathrm{n}(\%)\end{array}$ & $P$ Value & $\begin{array}{c}\text { Singlets } \\
\mathrm{n}=114 \\
\mathrm{n}(\%)\end{array}$ & $P$ Value & $\begin{array}{c}\text { TKI } \\
\mathrm{n}=395 \\
\mathrm{n}(\%)\end{array}$ & $P$ Value \\
\hline Acute myocardial infarction & 39 (3.6) & 3 (1.1) & 0.030 & $\begin{array}{ll}0 & (0.0)\end{array}$ & 0.235 & 3 (1.8) & 0.102 & $2 \quad(1.8)$ & 0.150 & 7 (1.8) & 0.075 \\
\hline Anemia & $480(44.1)$ & $121(43.4)$ & 0.822 & $17(44.7)$ & 0.940 & $65(38.9)$ & 0.207 & $42(36.8)$ & 0.136 & $107(27.1)$ & $<0.001$ \\
\hline Angina & $35(3.2)$ & $10(3.6)$ & 0.759 & $0 \quad(0.0)$ & 0.261 & $3(1.8)$ & 0.134 & $3(2.6)$ & 0.223 & $7 \quad(1.8)$ & 0.138 \\
\hline Bleeding events & $220(20.2)$ & $57(20.4)$ & 0.938 & $9(23.7)$ & 0.602 & $42(25.2)$ & 0.145 & $19(16.7)$ & 0.366 & $78(19.8)$ & 0.841 \\
\hline Bone marrow depression & $234(21.5)$ & $51(18.3)$ & 0.236 & $3 \quad(7.9)$ & 0.043 & $35(21.0)$ & 0.872 & $26(22.8)$ & 0.749 & $40(10.1)$ & $<0.001$ \\
\hline Cerebrovascular stroke & $43(4.0)$ & $13(4.7)$ & 0.595 & $1 \quad(2.6)$ & 0.343 & $8 \quad(4.8)$ & 0.610 & $4 \quad(3.5)$ & 0.202 & $21 \quad(5.3)$ & 0.253 \\
\hline Deep vein thrombosis & $7(0.6)$ & $2(0.7)$ & 0.892 & $0 \quad(0.0)$ & 0.786 & $0 \quad(0.0)$ & 0.367 & $0 \quad(0.0)$ & 0.497 & $0 \quad(0.0)$ & 0.114 \\
\hline Delay in wound healing & $4(0.4)$ & $0 \quad(0.0)$ & 0.401 & $0 \quad(0.0)$ & 0.872 & $2(1.2)$ & 0.150 & $0 \quad(0.0)$ & 0.671 & $2(0.5)$ & 0.309 \\
\hline Diarrhea & $156(14.3)$ & $43(15.4)$ & 0.650 & $7(18.4)$ & 0.482 & $22(13.2)$ & 0.688 & $15(13.2)$ & 0.731 & $71(18.0)$ & 0.086 \\
\hline Fistula & $4(0.4)$ & $1 \quad(0.1)$ & 0.410 & $0 \quad(0.0)$ & 0.872 & $2(1.2)$ & 0.150 & $0 \quad(0.0)$ & 0.671 & $1 \quad(0.3)$ & 0.386 \\
\hline Gastrointestinal perforations & $1 \quad(0.1)$ & $0(0.00)$ & 0.796 & $0 \quad(0.0)$ & 0.966 & $1(0.6)$ & 0.231 & $0 \quad(0.0)$ & 0.905 & $0 \quad(0.0)$ & 0.734 \\
\hline Heart failure & $101 \quad(9.3)$ & $22(7.9)$ & 0.467 & $3 \quad(7.9)$ & 0.227 & $10(6.0)$ & 0.163 & $16(14.0)$ & 0.103 & $36 \quad(9.1)$ & 0.921 \\
\hline Hepatotoxicity & $17 \quad(1.6)$ & $8 \quad(2.9)$ & 0.147 & $1 \quad(2.6)$ & 0.343 & $4 \quad(2.4)$ & 0.167 & $5 \quad(4.4)$ & 0.036 & $11(2.8)$ & 0.126 \\
\hline Leukopenia & $52(4.8)$ & $13 \quad(4.7)$ & 0.933 & $1 \quad(2.6)$ & 0.302 & $8 \quad(4.8)$ & 0.995 & $4 \quad(3.5)$ & 0.169 & $5 \quad(1.3)$ & 0.002 \\
\hline Neutropenia & $171(15.7)$ & $31(11.1)$ & 0.053 & $6(15.8)$ & 0.990 & $29(17.4)$ & 0.588 & $17(14.9)$ & 0.822 & $21 \quad(5.3)$ & $<0.001$ \\
\hline Peripheral neuropathy & 14 (1.3) & $5(1.8)$ & 0.520 & $0 \quad(0.0)$ & 0.617 & $3(1.8)$ & 0.219 & $1 \quad(0.9)$ & 0.354 & $2(0.5)$ & 0.198 \\
\hline Pruritus, skin rash & $98 \quad(9.0)$ & $26 \quad(9.3)$ & 0.872 & $3 \quad(7.9)$ & 0.230 & $21(12.6)$ & 0.143 & $12(10.5)$ & 0.593 & $59(14.9)$ & 0.001 \\
\hline Pulmonary embolism & $83 \quad(7.6)$ & $38(13.6)$ & 0.002 & $3 \quad(7.9)$ & 0.237 & $13(7.8)$ & 0.944 & $10 \quad(8.8)$ & 0.664 & $26 \quad(6.6)$ & 0.495 \\
\hline Thrombocytopenia & $135(12.4)$ & $27 \quad(9.7)$ & 0.208 & $3 \quad(7.9)$ & 0.160 & $24(14.4)$ & 0.478 & $10 \quad(8.8)$ & 0.257 & $19 \quad(4.8)$ & $<0.001$ \\
\hline Transient cerebral ischemia & $7(0.6)$ & $1 \quad(0.4)$ & 0.578 & $0 \quad(0.0)$ & 0.786 & $4 \quad(2.4)$ & 0.038 & $1 \quad(0.9)$ & 0.379 & $3(0.8)$ & 0.260 \\
\hline Venous or arterial thrombosis & $226(20.7)$ & $64(22.9)$ & 0.430 & $6(15.8)$ & 0.455 & $31(18.6)$ & 0.510 & $21(18.4)$ & 0.555 & $63(16.0)$ & 0.038 \\
\hline Visual disturbance & $36 \quad(3.3)$ & $5(1.8)$ & 0.185 & $1 \quad(2.6)$ & 0.367 & $3(1.8)$ & 0.125 & $2(1.8)$ & 0.174 & $8 \quad(2.0)$ & 0.064 \\
\hline Any adverse event & $833(76.6)$ & $220(78.9)$ & 0.417 & $27(71.1)$ & 0.432 & $130(77.8)$ & 0.715 & $81(71.1)$ & 0.189 & $262(66.3)$ & $<0.001$ \\
\hline
\end{tabular}

platinum doublets of either carboplatin + paclitaxel or carboplatin + pemetrexed improve overall survival in those patients aged 70 years and older who had a performance status of 2 , compared with single-agent treatments. ${ }^{20,21}$ We found similar results for patients using single agents. Singlets had the lowest median months of survival, and in all models, singlets were associated with reduced survival compared with platinum doublets.

Patients taking TKIs had the lowest rate of adverse events. However, the TKI group had a higher rate of skin rash. The most common adverse events from the package insert (>20\%) associated with erlotinib are rash-like events and diarrhea. ${ }^{22}$ Pemetrexed-based regimens were associated with a higher rate of pulmonary embolism compared with platinum doublets.

\section{Limitations}

This study has several limitations. Selection bias is a possible source of confounding in retrospective studies. Patients may receive one systemic first-line therapy over another based on performance status and other patient characteristics or according to prescriber preferences. We were not able to include performance status, but we did include the Deyo modification of the Charlson comorbidity score and other covariates to act as a proxy for performance status in our propensity score models. While we had enough sample size for the platinum doublet and TKI groups, other treatment groups, especially the pemetrexed + bevacizumab-based group and the singlets group, did not reach adequate sample size. We, therefore, cannot exclude the possibility that a larger sample would produce different results.

Other limitations include the lack of information on molecular biomarker status (EGFR or ALK), severity of adverse events, weight loss, disease progression, tumor response, radiotherapy, surgical procedures, and whether the patient was enrolled in a clinical trial; if available, information in these areas could potentially affect the choice of and response to first-line therapy. This study did not assess therapy duration and dosing of systemic therapies; in addition, we were not able to capture information on the more subjective measures such as pain and quality of life. Other than subsequent therapy for NSCLC, we did not include other treatments or medications (e.g., drugs for pain or steroids) that were given to patients at the same time or after first-line therapy. Finally, all data were obtained from a single managed care organization in California, so the results might not be generalizable to other settings or uninsured populations. 
The treatment of NSCLC has rapidly evolved over the past few years. The addition of targeted therapies to standard chemotherapy and the approval of immune checkpoint inhibitors such as nivolumab, atezolizumab, and pembrolizumab represent important milestones in the treatment of NSCLC. Because we used data only through 2014, we were not able to include newer therapies. Survival outcomes and analyses around the potentially serious complications associated with newer therapies are important areas for future comparative effectiveness research.

\section{Conclusions}

This study explored treatment patterns and overall survival for first-line systemic therapies in patients with advanced NSCLC in a large, integrated health care system. These results provide insight on the types of therapies being prescribed to patients with advanced stage NSCLC and factors associated with overall survival between these therapies.

In this cohort of patients with advanced NSCLC, most patients received a platinum agent with or without bevacizumab or pemetrexed, a TKI such as erlotinib, or a single agent such as gemcitabine. Younger patients (aged $<65$ years) using bevacizumab + pemetrexed-based combinations had a survival advantage over those using platinum doublets, and this finding merits further investigation. Younger patients using TKIs also had a survival advantage, and TKIs were associated with fewer adverse events. Compared with platinum doublets, we found no survival advantage for older patients using bevacizumab or pemetrexed, which suggests that combination therapy of a platinum agent and taxane, such as carboplatin and paclitaxel, is a reasonable option for older patients who are not candidates for targeted therapy.

\section{Authors}

MICHELE M. SPENCE, PhD, Pharmacy Outcomes Research Group; JENNIFER T. CHANG, PharmD, MPH, Drug Information Services; MIRTA MILLARES, PharmD, FCSHP, FASHP, Drug Information Services and Pharmacy Outcomes Research; and NAZIA RASHID, PharmD, MS, Pharmacy Outcomes Research Group, Kaiser Permanente Southern California, Downey, California. RITA L. HUI, PharmD, MS, Pharmacy Outcomes Research Group, Kaiser Permanente Northern California, Oakland, California, and JOANNE E. SCHOTTINGER, MD, Quality and Clinical Analysis, Kaiser Permanente Southern California, Pasadena, California.

AUTHOR CORRESPONDENCE: Michele M. Spence, PhD, Clinical Pharmacy Research Scientist, Pharmacy Outcomes Research Group, Kaiser Permanente Southern California, 12254 Bellflower Blvd., Downey, CA 90242. Tel.: 562.658.3528; E-mail: michele.m.spence@kp.org.

\section{DISCLOSURES}

No outside funding supported this study. Rashid has received past funding from Bristol-Myers Squibb, Astellas, Novartis, and Pfizer. No other authors report any potential financial conflicts of interest.

Study concept and design were primarily contributed by Spence and Hui, with input from the other authors. Hui, Spence, and Rashid took the lead in data collection, and data interpretation was performed by Schottinger, Millares, and Spence, assisted by the other authors. The manuscript was written primarily by Spence, along with Chang, and revised by Spence, with input from the other authors.

\section{ACKNOWLEDGMENTS}

The authors thank Fang Niu, MS, for statistical consultation and advice, and Tim Chiu, PharmD, BCPS, for clinical information and advice.

\section{REFERENCES}

1. National Cancer Institute. SEER stat fact sheets: lung and bronchus cancer. Available at: http://seer.cancer.gov/statfacts/html/lungb.html. Accessed December 22, 2016.

2. Dela Cruz CS, Tanoue LT, Matthay RA. Lung cancer: epidemiology, etiology, and prevention. Clin Chest Med. 2011;32(4):605-44.

3. Sandler A, Gray R, Perry MC, et al. Paclitaxel-carboplatin alone or with bevacizumab for non-small-cell lung cancer. N Engl J Med. 2006;355(24):2542-50.

4. Ramalingam SS, Dahlberg SE, Langer CJ, et al. Outcomes for elderly, advanced-stage non small-cell lung cancer patients treated with bevacizumab in combination with carboplatin and paclitaxel: analysis of Eastern Cooperative Oncology Group trial 4599. J Clin Oncol. 2008;26(1):60-65.

5. Zhu J, Sharma DB, Gray SW, et al. Carboplatin and paclitaxel with vs without bevacizumab in older patients with advanced non-small cell lung cancer. JAMA. 2012;307(15):1593-601.

6. Ritzwoller DP, Carroll NM, Delate T, et al. Comparative effectiveness of adjunctive bevacizumab for advanced lung cancer: the cancer research network experience. J Thorac Oncol. 2014;9(5):692-701.

7. Langer C, Ravelo A, Hazard SJ, et al. Comparison of survival and hospitalization rates between Medicare patients with advance NSCLC treated with bevacizumab-carboplatin-paclitaxel and carboplatin-paclitaxel: a retrospective cohort study. Lung Cancer. 2014;86(3):350-57.

8. Langer CJ, Socinski MA, Patel JD, et al. Isolating the role of bevacizumab in elderly patients with previously untreated nonsquamous non-small cell lung cancer: secondary analyses of the ECOG 4599 and PointBreak Trials. Am J Clin Oncol. 2016;39(5):441-47.

9. National Comprehensive Cancer Network. Clinical Practice Guidelines in Oncology. Non-small cell lung cancer. Version 4.2106. Available at: https:// www.nccn.org/professionals/physician_gls/f_guidelines.asp. Accessed December 22, 2016.

10. Scagliotti GV, Parikh P, von Pawel J, et al. Phase III study comparing cisplatin plus gemcitabine with cisplatin plus pemetrexed in chemotherapynaïve patients with advanced-stage non-small-cell lung cancer. J Clin Oncol. 2008;26(21):3543-51

11. Kaiser Permanente. Fast facts about Kaiser Permanente. Available at: http://share.kaiserpermanente.org/article/fast-facts-about-kaiser-permanente. Accessed December 22, 2016.

12. Klabunde CN, Legler JM, Warren JL, Baldwin LM, Schrag D. A refined comorbidity measurement algorithm for claims-based studies of breast, prostate, colorectal, and lung cancer patients. Ann Epidemiol. 2007;17(8):584-90.

13. Rajeswaran A, Trojan A, Burnand B, Giannelli M. Efficacy and side effects of cisplatin- and carboplatin-based doublet chemotherapeutic regimens versus non-platinum-based doublet chemotherapeutic regimens as first line treatment of metastatic non-small cell lung carcinoma: a systematic review of randomized controlled trials. Lung Cancer. 2008;59(1):1-11. 


\section{Treatment Patterns and Overall Survival Associated with First-Line Systemic Therapy for Patients with Advanced Non-Small Cell Lung Cancer}

14. Zornosa C, Vandergrift JL, Kalemkerian GP, et al. First-line system therapy practice patterns and concordance with NCCN guidelines for patients diagnosed with metastatic NSCLC treated at NCCN institutions. J Natl Compr Canc Netw. 2012;10(7):846-56.

15. Paik PK, Varghese AM, Sima CS, et al. Response to erlotinib in patients with EGFR mutant advanced non-small cell lung cancers with a squamous or squamous-like component. Mol Cancer Ther. 2012;11(11):2535-40.

16. Midha A, Dearden S, McCormack R. EGFR mutation incidence in onsmall-cell lung cancer of adenocarcinoma histology: a systematic review and global map by ethnicity (mutMapII). Am J Cancer Res. 2015;5(9):2892-911.

17. Patel JD, Socinski MA, Garon EB, et al. PointBreak: a randomized phase III study of pemetrexed plus carboplatin and bevacizumab followed by maintenance pemetrexed and bevacizumab versus paclitaxel plus carboplatin and bevacizumab followed by maintenance bevacizumab in patients with stage IIIB or IV nonsquamous non-small cell lung cancer. J Clin Oncol. 2013;31(34):4349-57.
18. Barlesi F, Scherpereel A, Rittmeywr A, et al. Randomized phase III trial of maintenance bevacizumab with or without pemetrexed after first-line induction with bevacizumab, cisplatin, and pemetrexed in advanced nonsquamous non-small cell lung cancer: AVAPERL. J Clin Oncol. 2013;31(24):3004-11.

19. Patel JD, Hensing TA, Rademaker A, et al. Phase II study of pemetrexed and carboplatin plus bevacizumab with maintenance pemetrexed and bevacizumab as first-line therapy for nonsquamous non-small-cell lung cancer. J Clin Oncol. 2009;27(20);3284-89.

20. Quoix E, Zalcman G, Oster JP, et al. Carboplatin and weekly paclitaxel doublet chemotherapy compared with monotherapy in elderly patients with advanced non-small-cell lung cancer: IFCT-0501 randomised, phase 2 trial. Lancet. 2011;378(9796):1079-88.

21. Zukin M, Barrios CH, Pereira JR, et al. Randomized phase III trial of single-agent pemetrexed vuersus carboplatin and pemetrexed in patients with advanced non-small-cell lung cancer and Eastern Cooperative Oncology Group performance status of 2. J Clin Oncol. 2013;31(23):2849-53.

22. TARCEVA (erlotinib) tablets, for oral use. OSI Pharmaceuticals and Genentech. Revised October 2016. Available at: https://www.gene.com/ download/pdf/tarceva_prescribing.pdf. Accessed December 22, 2016. 


\section{APPENDIX A Description of 6 Mutually Exclusive Chemotherapy Groups}

\section{Chemotherapy Group, N (\% of 2,081 in Cohort)}

Doublets, 1,088 (52.3)

\section{Regimen}

Carboplatin + paclitaxel

Carboplatin + gemcitabine

Cisplatin + etoposide

Cisplatin + gemcitabine

Cisplatin + vinorelbine

Carboplatin + pemetrexed

Cisplatin + pemetrexed

Pemetrexed

Pemetrexed + bevacizumab-based combinations, $38(1.8)$

Bevacizumab-based regimens,

$167(8.0)$

Singlets, $114(5.5)$

Tyrosine-kinase inhibitors, 395 (19.0)
Bevacizumab + carboplatin + pemetrexed

Bevacizumab + cisplatin + pemetrexed

Bevacizumab + pemetrexed

Bevacizumab + carboplatin + paclitaxel

Bevacizumab + carboplatin + gencitabine

Bevacizumab

Bevacizumab + cisplatin + gemcitabine

Bevacizumab + gemcitabine

Gemcitabine

Vinorelbine

Paclitaxel

Docetaxel

Erlotinib
Number of Patients in Regimen (\% of Chemotherapy Group)

\begin{tabular}{rr}
739 & $(67.9)$ \\
171 & $(15.7)$ \\
103 & $(9.5)$ \\
47 & $(4.3)$ \\
28 & $(2.6)$ \\
155 & $(55.6)$ \\
83 & $(29.7)$ \\
41 & $(14.7)$ \\
21 & $(55.3)$ \\
13 & $(34.2)$ \\
4 & $(10.5)$ \\
140 & $(83.8)$ \\
13 & $(7.8)$ \\
10 & $(6.0)$ \\
2 & $(1.2)$ \\
2 & $(1.2)$ \\
\hline 38 & $(33.3)$ \\
37 & $(32.5)$ \\
28 & $(24.6)$ \\
11 & $(9.6)$ \\
395 & $(100.0)$ \\
\hline
\end{tabular}

\section{APPENDIX B Adverse Events with Corresponding ICD-9-CM Diagnosis and Diagnosis-Related Group Codes}

\begin{tabular}{|c|c|c|}
\hline Diagnosis/Adverse Event & DRG Codes & ICD-9-CM Diagnosis Codes \\
\hline Acute myocardial infarction & $121-123,280-285$ & $410 . x x$ \\
\hline Anemia & $808,809,810$ & $283.0,283.9,285.22,285.3,285.9$ \\
\hline Angina & 140,311 & $411.1,411.8,413 . \mathrm{xx}$ \\
\hline Bleeding events & & $\begin{array}{l}360.43,362.81,364.41,430,459.0,511.89,569.3,578,578.1,599.7, \\
623.8,626.2,626.6,784.7,786.3\end{array}$ \\
\hline Bone marrow depression & $574,808-810$ & $284.11,284.12,284.19,284.9$ \\
\hline Cerebrovascular stroke & $014,015,559,061-068$ & $430 . x x-432 . x x$ \\
\hline Deep vein thrombosis & $128,294,295$ & $451.1,451.2,451.81,453.2$ \\
\hline Delay in wound healing & & $872-897$ \\
\hline Diarrhea & $182-184,391,392$ & 787.91 \\
\hline Fistulas & & $510.0,530.84,576.4,593.89,596.2,619.8$ \\
\hline Gastrointestinal perforations & $174-177,569,570,330,331,377-382$ & $\begin{array}{l}530.4,531.1,531.2,531.5,531.6,532.1,532.2,532.5,532.6,533.1, \\
533.2,533.5,533.6,534.1,534.2,534.5,534.6,569.83\end{array}$ \\
\hline Heart failure & $127,291,292,293$ & 428 \\
\hline Hepatotoxicity & $205,206,441-443$ & $570,794.8,864.0$ \\
\hline Leukopenia & $398,399,814-816$ & 288.5 \\
\hline Neutropenia & & $288.0,288.9,780.61$ \\
\hline Peripheral neuropathy & $018,019,073,074$ & $337,357.89$ \\
\hline Pruritus, skin rash & $283,284,606,607$ & $698.9,782.1$ \\
\hline Pulmonary embolism & $078,175,176$ & 415.1 \\
\hline Thrombocytopenia & 397,813 & $287.49,287.5$ \\
\hline Transient cerebral ischemia & 524,069 & 435,437 \\
\hline Venous or arterial thrombosis & $130,131,299,300,301$ & $\begin{array}{l}250.7,440.0,440.2,440.3,440.8,440.9,441,442.0,442.2,442.3 \\
442.8,442.9,443.1,443.2,443.8,443.9,444,445,447.0,447.1 \\
447.2,447.5,447.8,447.9,448.0,448.9,451.0,451.8,451.9,453.1 \\
453.4,453.8,453.9,454,456.3,456.8,459.1,459.2,459.81,785.4 \\
908.3,908.4,997.2,997.79\end{array}$ \\
\hline Visual disturbance & $043,046-048,124,125$ & $368.1,368.2,368.3,368.8,368.9$ \\
\hline
\end{tabular}

\footnotetext{
DRG = diagnosis-related group; ICD-9-CM = International Classification of Diseases, Ninth Revision, Clinical Modification.
} 